\title{
TOEPLITZ OPERATORS ON CARTAN DOMAINS ESSENTIALLY COMMUTE WITH A BILATERAL SHIFT
}

\author{
MIROSLAV ENGLIŠ
}

(Communicated by Theodore W. Gamelin)

\begin{abstract}
For bounded symmetric domains $\Omega \subset \mathbf{C}^{N}$, a bilaterial shift operator $U$ is shown to exist on the Bergman space $A^{2}(\Omega)$ such that $U T_{f}-T_{f} U$ is a compact operator for all Toeplitz operators $T_{f}$. This may be viewed as an extension of the well-known fact that $S^{*} T S-T=0$ whenever $T$ is a Toeplitz operator on $H^{2}, S$ being the unilateral shift. It also follows that the $C^{*}$-algebra generated by Toeplitz operators on $A^{2}(\Omega)$ does not contain all bounded operators.
\end{abstract}

Let $\Omega$ be a bounded symmetric (Cartan) domain with its standard (HarishChandra) realization in $\mathbf{C}^{N}$ [6], $d v$ the $2 n$-dimensional Lebesgue measure on $\Omega$, and $L^{2}(\Omega, d v)$ the Hilbert space of square-integrable complex-valued functions on $\Omega$. The Bergman space, $A^{2}(\Omega)$, is the closed subspace of $L^{2}(\Omega, d v)$ consisting of functions analytic on $\Omega$. Denote by $P$ the orthogonal projection from $L^{2}$ onto $A^{2}$. For $f \in L^{\infty}(\Omega)$, the Toeplitz operator $T_{f}: A^{2} \rightarrow A^{2}$ and the Hankel operator $H_{f}: A^{2} \rightarrow L^{2} \ominus A^{2}$ are given by

$$
T_{f} x=P(f x), \quad H_{f} x=(I-P)(f x) .
$$

These operators generalize, in an obvious way, the well-known Toeplitz and Hankel operators on $H^{2}$ [5, Chapter 25].

The main result of the present note is the following theorem.

Theorem. There is a bilateral shift $U$ on $A^{2}(\Omega)$ such that for all $f \in L^{\infty}(\Omega)$ the commutator $\left[U, T_{f}\right] \equiv U T_{f}-T_{f} U$ is a compact operator.

Proof. We first introduce some notation and terminology from [1, 2]. Let $\beta(\cdot, \cdot)$ be the Bergman metric on $\Omega[6]$ and $\operatorname{dist}(\cdot, \cdot)$ the usual euclidean metric in $\mathbf{C}^{N}=\mathbf{R}^{2 N}$. Denote by $\operatorname{BC}(\Omega)$ the algebra of bounded continuous complex-valued functions on $\Omega$, with $C_{\partial}(\Omega)$ the subalgebra of functions for which $f(z) \rightarrow 0$ as $\operatorname{dist}(z, \partial \Omega) \rightarrow 0$. Define

$$
\operatorname{Osc}(f, z)=\sup \{|f(w)-f(z)|: \beta(z, w)<1\},
$$

the oscillation of $f$ at $z$, and

$$
\operatorname{VO}_{\partial}(\Omega)=\left\{f \in \operatorname{BC}(\Omega): \operatorname{Osc}(f, \cdot) \in C_{\partial}(\Omega)\right\},
$$

Received by the editors May 30, 1991.

1991 Mathematics Subject Classification. Primary 47B35; Secondary 32M15.

Key words and phrases. Toeplitz operators, bounded symmetric domains. 
the algebra of functions with vanishing oscillation at the boundary.

Consider the function $\Phi$ on $\Omega$ given by

$$
\Phi(z)=\exp (i \sqrt{\beta(0, z)}) .
$$

It is well known [1] that $\Phi \in \mathrm{VO}_{\partial}(\Omega)$. Indeed,

$$
\begin{aligned}
|\Phi(z)-\Phi(w)| & \leq\left|\beta(0, z)^{1 / 2}-\beta(0, w)^{1 / 2}\right| \\
& \leq \frac{|\beta(0, z)-\beta(0, w)|}{\beta(0, z)^{1 / 2}+\beta(0, w)^{1 / 2}} \\
& \leq \frac{\beta(z, w)}{\beta(0, z)^{1 / 2}+\beta(0, w)^{1 / 2}}
\end{aligned}
$$

and the assertion is immediate since $\beta(0, x) \rightarrow+\infty$ as $\operatorname{dist}(x, \partial \Omega) \rightarrow 0$. Hence, by [2, Theorem B], $H_{\Phi}$ and $H_{\bar{\Phi}}$ are compact operators. According to a well-known commutator identity for Toeplitz operators,

$$
T_{f} T_{g}-T_{g} T_{f}=H_{g}^{*} H_{f}-H_{\frac{*}{f}}^{*} H_{g}
$$

for arbitrary $f, g \in L^{\infty}(\Omega)$. Taking $g=\Phi$, we see that the commutator $T_{f} T_{\Phi}-T_{\Phi} T_{f} \equiv\left[T_{f}, T_{\Phi}\right]$ is compact $\forall f \in L^{\infty}(\Omega)$. Thus the proof will be accomplished if we find a compact operator $K$ such that $T_{\Phi}+K$ is a bilateral shift with respect to some basis. Since, in particular, $\left[T_{\bar{\Phi}}, T_{\Phi}\right]$ is compact, $T_{\Phi}$ is essentially normal. Hence, by the Brown-Douglas-Fillmore theory [3], it suffices to show that the essential spectrum of $T_{\Phi}$ is the unit circle, $T$, and that ind $T_{\Phi}=0$.

Denote

$$
\mathscr{Q}=\left\{f \in L^{\infty}(\Omega): H_{f} \text { and } H_{\bar{f}} \text { are compact }\right\},
$$

and let $\tau(\mathscr{Q})$ be the $C^{*}$-algebra generated by $\left\{T_{f}: f \in \mathscr{Q}\right\}$. By [1, Theorem $\mathrm{B}]$, there is a $C^{*}$-isomorphism

$$
\tau(\mathscr{Q}) / \text { Compacts } \simeq \mathrm{VO}_{\partial} / C_{\partial}(\Omega)
$$

which maps $T_{f}$ into the coset $[f]$ of $f$ in $\mathrm{VO}_{\partial} / C_{\partial}$. It follows that, for $f \in \mathscr{Q}, \sigma_{e}\left(T_{f}\right)$ coincides with the spectrum of $[f]$ in $\mathrm{VO}_{\partial} / C_{\partial}(\Omega)$. Since $[f]$ is invertible in $\mathrm{VO}_{\partial} / C_{\partial}$ iff it is invertible in $L^{\infty} / C_{\partial}$, the latter spectrum is easily seen to coincide with the set of all cluster values of $f$ at $\partial \Omega$,

$$
\sigma_{\mathrm{VO}_{\partial} / C_{\partial}}([f])=\bigcap_{R>0} \overline{\{f(z): \beta(0, z)>R\}} .
$$

Taking $f=\Phi$, we conclude that

$$
\sigma_{e}\left(T_{\Phi}\right)=\bigcap_{R>0} \overline{\{\Phi(z): \beta(0, z)>R\}}=\mathbf{T},
$$

which proves the first claim.

To compute the Fredholm index of $T_{\Phi}$, consider the functions

$$
\Phi_{m}(z)=\exp \left(\frac{i}{m} \sqrt{\beta(0, z)}\right),
$$

where $m$ is a positive integer (thus, $\Phi_{1}=\Phi$ ). Everything that was said about $\Phi$ is readily seen to apply to $\Phi_{m}$ as well: $\Phi_{m}$ is a bounded continuous function that belongs to $\mathrm{VO}_{\partial}$, so that $H_{\Phi_{m}}, H_{\overline{\Phi_{m}}}$ are compact operators and $\Phi_{m} \in \mathscr{Q}$; 
further, $\sigma_{e}\left(T_{\Phi_{m}}\right)=\mathbf{T}$, so $T_{\Phi_{m}}$ are Fredholm operators. Since $\Phi_{m}^{m}=\Phi$, it follows from $(*)$ that $T_{\Phi_{m}}^{m}=T_{\Phi}$ modulo the compacts. Thus, we have

$$
\text { ind } T_{\boldsymbol{\Phi}}=\text { ind } T_{\Phi_{m}}^{m}=m \cdot \text { ind } T_{\Phi_{m}}
$$

for all positive integers $m$, which is only possible when ind $T_{\Phi}=0$. The proof is complete.

For Toeplitz operators on the $N$-dimensional Fock space $A^{2}\left(\mathbf{C}^{N}\right), N \geq 1$, a similar result was obtained by the present author in [4]. It is also shown there that for arbitrary bounded planar domain $\Omega \subset \mathbf{C}$ there exists a unilateral shift operator $S$ on the Bergman space $A^{2}(\Omega)$ such that, for all Toeplitz operators $T_{f}, S T_{f}-T_{f} S$ is compact. The assertion was also proved for the one-dimensional Fock space $A^{2}(\mathbf{C})$. These results may be compared with the classical characterization [5, Problem 242] of Toeplitz operators on $H^{2}$ :

$$
T \text { is a Toeplitz operator } \Leftrightarrow S^{*} T S=T,
$$

$S$ being the unilateral shift on $H^{2}$.

As a direct consequence of the above theorem, we have

Corollary. The $C^{*}$-algebra generated by all Toeplitz operators $\left\{T_{f}: f \in L^{\infty}(\Omega)\right\}$, where $\Omega$ is a bounded symmetric domain in $\mathbf{C}^{N}$, does not contain all bounded operators on $A^{2}(\Omega)$.

Proof. Let $U=T_{\Phi}+K$ be the bilateral shift obtained above. Since

$$
\begin{gathered}
{[A+B, U]=[A, U]+[B, U], \quad[c A, U]=c[A, U],} \\
{\left[A^{*}, U\right]=U[A, U]^{*} U, \quad[A B, U]=A[B, U]+[A, U] B,}
\end{gathered}
$$

and

$$
\left\|A_{n}-A\right\| \rightarrow 0 \text { implies }\left\|\left[A_{n}, U\right]-[A, U]\right\| \rightarrow 0,
$$

we see that the essential commutant, $U^{\text {'ess }}$, of $U$ is a $C^{*}$-algebra. According to the preceding theorem, it contains all operators $T_{f}, f \in L^{\infty}(\Omega)$. It follows that $U^{\prime \text { ess }}$ contains the $C^{*}$-algebra generated by them as well, and we only have to find an operator not belonging to $U^{\prime \text { ess }}$. Let $\left\{e_{n}\right\}_{n \in Z}$ be a basis with respect to which $U$ is the bilateral shift, i.e.,

$$
U e_{n}=e_{n+1}, \quad n \in \mathbf{Z},
$$

and let $J$ be given by

$$
J e_{n}=(-1)^{n} e_{n}, \quad n \in \mathbf{Z} .
$$

Then $J U-U J=2 J U$ is not compact, and so $J \notin U^{\prime e s s}$.

\section{REFERENCES}

1. C. A. Berger, L. A. Coburn, and K. H. Zhu, Function theory on Cartan domains and the Berezin-Toeplitz symbol calculus, Amer. J. Math. 110 (1988), 921-953.

2. D. Békollé, C. A. Berger, L. A. Coburn, and K. H. Zhu, BMO in the Bergman metric on bounded symmetric domains, J. Funct. Anal. 93 (1990), 310-350.

3. L. G. Brown, R. G. Douglas, and P. A. Fillmore, Unitary equivalence modulo the compact operators and extensions of $C^{*}$-algebras, Lecture Notes in Math., vol. 345, Springer-Verlag, Berlin and Heidelberg, 1973, pp. 58-128. 
4. M. Engliš, Density of algebras generated by Toeplitz operators on Bergman spaces, Ark. Mat. (to appear).

5. P. R. Halmos, A Hilbert space problem book, Graduate Texts in Math., Springer-Verlag, New York, 1967.

6. S. Helgason, Differential geometry, Lie groups and symmetric spaces, Academic Press, New York, 1978.

Mathematical Institute, Czechoslovak Academy of Sciences, Žitná 25, 11567 Prague 1, Czechoslovakia

E-mail address: englis@cspgas11.bitnet

Current address: Department of Mathematics, Kansas State University, Manhattan, Kansas 66506

E-mail address: englis@ksuvm.ksu.edu 